Pediat. Res. 5: 711-718 (1971)

\title{
Index to Volume 5
}

(Numbers followed by asterisk refer to Society Abstracts)

Aagenaes, O. 93*

Abaci, F. 393*

Abdo-Bassols, F. 213

Abranison, D. $396^{*}$

$\beta$-D- $N$-acetylglucosaminidase 366

Acid-basc homeostasis 345,523

Acidosis 213

Adam, P. 417*

$\Lambda$ ddanki, S. 421*

Addo, N. 329

Adenine 77

$S$-Adenosylmethionine 360

Adenyl cyclase 126

Ader, R. 638*

Adler, W. H., III 379*

Admirand, W. H. 391*

Adrianzen T., B. 691

Airway closure 10

Airway resistance 10

Al-Aish, M. S. 501

Aladjem, S. 4l1*

Alakija, W. 388*

Albritton, W. L. $414^{*}$

Aicade, E. 579

Niden, E. R. 429*

Aldosterone 618, 626

Alkaline phosphatase $95,{ }^{*} 704$

Alkalosis 618

Alkjacrsig, N. 655*

Allderdice, P. W. 423, * 424*

Allen, $\Lambda$. C. 345

Allen, E. 403*

Altemeier, W. A., III $379^{*}$

Altman, Y. 87*

Ames, M. 374*

Amino acid metabolism 478

Amino acids 33, 173, 329, 698

Aminoaciduria 591

Ammonium 345

Ammiocentesis 366

Amos, D. B. $379^{*}$

Anagnostakis, D. 87*

Anania, R. 385*

Anast, C. S. 668

Anderson, G. 426*

Ando, T. 478

Angiotcnsin 626

Anoxia 173

Antiplasmin 206

$\alpha$-I-Antitrypsin 206

Apell, G. 493
Appleman, H. D. $390^{*}$

Arcilla, M. B. $422^{*}$

Arcilla, R. A. $428^{*}$

Argucdas G, J. A. 402*

Arnaud, C. 392*

Arrobio, J. O. $649^{*}$

Arylsulfatasc $A 366$

Asphyxia 452

Astaldi, G. 96*

Atkins, T. E. $376^{*}$

Atwood, G. F. 425*

August, C. S. 539

Auld, P. A. M. 10, 428*

Aur, R. J. 408*

Auricchio, S. 84*

Aurich, G. 88*

Avery, M. E. 416*

Aylsworth, A. 652*

Ayoub, E. M. 379, * $405^{*}$

Bacolla, D. 93*

Baehner, R. L. 408*

Baerlocher, K. 382*

Baghdassarian, A. 398*

Baker, I.. 396*

Banerjec, S. D. 653*

Barbero, G. J. 390*

Barnctt, T. B. 427*

Barrett, C. T. 452

Bartcls, H. 95*

Bartlett, J. 40.5*

Bartter, F. C. 626

Battaglia, F. C. $419, * 640 *$

Baum, D. 395*

Bazaral, M. 381*

Beagles 193

Bchavioral patterns 298

Behrman, R. E. 415*

Bellanti, J. A. 380*

Beran, A. V. 199

Berezesky, I. K. 501

Bergman, G. 390*

Bergstrom, W. H. 399*

Berk, P. D. 256

Bcrkel, A. I. 378, 539

Berlin, N. I. 256

Bernal, A. 417*

Bernfield, M. R. 653*

Betten, J. 425*

Bicarbonatc 345

Biglieri, E. G. $400^{*}$
Bile 704

Bile acids 274

Bilirubin 256, 548, 683, 704

Binnington, V. 137

Birch, H. G. 579

Bishop, A. J. 113

Biundo, M. C. $380^{*}$

Blackburn, M. G. 4.12*

Blacse, R. M. 378*

Blanc, W. A. 17, 374*

Blizzard, R. M. 53, 398*

Blood-brain barricr 356

Blood flow 199, 452

Blood pH 213

Blood pressure 472

Blood volume 523

Bloom, R. S. 428*

Bloomer, J. R. 256

Boat, T. F. 626

Bockman, D. E. 377*

Bodegård, G. 90*

Bodenbender, J. G. 421*

Body composition 523, 673

Body weight 193

Bonforte, R. J. 378*

Bongiovanni, A. M. 389, *396, *398, * 400,* $423^{*}$

Boon, D. J. 390, * 644*

Borer, R. C., Jr. 415, * 655**

Borssén, R. 39*

Bottini, E. 96,* 423 *

Boucher, D. W. 403*

Bouttc, C. A. 407*

Bouver, N. G. 493

Bowie, E. J. W. 409*

Bradley, K. H. $50 \mathrm{I}$

Brady, J. 414*

Brain, 173, 199, 265, 329, 466, 659, 691

Brandt, C. D. 649 *

Brascl, J. A. 418, * 420*

Bratlid, D. 94*

Breg, W. R. 423, * 424*

Brickman, A. 403*

Brill, A. 373*

Briskcy, E.J. 281

Britt, S. 377*

Brodchl, J. 591

Broughton-Pipkin, F. 93*

Brown, A. K. 493

Brown, D. M. 181

Brown, R. S. 642* 
Brown fat 126

Bruck, E. 397*

Bruncll, P. A. 403*

Bryan, A. C. $426,{ }^{*} 428^{*}$

Bryan, M. H. 413*

Bryans, A. M. 137

Bryson, M. F. 385*

Bucci, G. 85*

Buckley, R. H. 379*

Buist, N. R. M. 287

Burgio, G. R. 88*

Burke, E. C. $383^{*}$

Burke, V. $84^{*}$

Burley, S. 405*

Burr, I. M. 397*

Burt, L. S. 374*

Butler, D. G. 389*

Buynak, E. B. 402*

Buzon, M. 386*

Calcagno, P. L. 335

Calcium 40, 181, 668

Calcitonin 181, 668

Campbell, A. G. M. 394*

Campbell, J. A. 151

Carbohydratc 698

Carbohydrate metabolism 213

Cardiac output 335,452

Cardiovascular system 472

Carey, J. B., Jr. 274

Carnclli, V. 97*

Cassady, G. 673

Cassens, R. G. 281

Cassin, S. 427*

Castcllanos, R. 586

Castells, S. 329

Castro, J. R. 4.08*

Catch-up growth 59, 691

Ciatecholamincs 360, 435

Catechol $O$-incthyltransferase 360

Cation permeability 159

Catz, C. S. $420^{*}$

Cell size 312

Ccll lysis 159

Ccrebral circulation 659

Chait, M. M. 373*

Chall, M. N. $376^{*}$

Chan, J. C. 395*

Chance, G. W. 413*

Chanock, R. M. 649*

Chase, H. P. 372, ${ }^{*} 375^{*}$

Check, D. B. 312, 418, ${ }^{*} 605$

Cheema, R. A. 407*

Chemotactic response 487

Chernay, P. R. 424*

Chernick, V. 113, 428,*654*

Chessels, J. M. 95*

Cheung, G. P. 372*

Ghildhood 579

Choffat, J. M. 91*

Christiansen, P. A. 698
Chromosomal aberration 64

Chua, L. 415*

Cintron, C. $398, * 423 *$

Circulation 193

Clark, S. H. 416*

Clausen, J. 85*

Clcments, R. S. 396*

Clow, C. 382,* 392*

Clyde, W. 405*

$\mathrm{CO}_{2} 213$

Cohen, M. I. 391, * 641*

Cohen, P. 392*

Cohlan, S. Q. 131

Cohn, J. 96*

Cole, B. R. 655*

Colle, E. 397*

Colombo, J. P. 97*

Colombo, U. 85*

Colten, H. R. 404*

Communication gap 298

Conen P. E. 407*

Cook, C. D. 384*

Cooke, R. E. 566

Cooper, C. C. 281

Cooper, H. A. 409*

Cooper, L. Z. 403*

Cooper, M. D. 377*

Corbcel, L. M. 83*

Cord blood 101, 493

Cornblath, M. $394, * 420,{ }^{*} 518$

Cornell, R. 386*

Corrigan, J. J., Jr. 404*

Costom, B. H. 654*

Corwin, R. M. 399*

Cowger, M. L. 419*

Cravioto, J. 579

Cretins 668

Crigler-Najjar syndrome 256

Crocker, J. F. S. 654*

Cudcrman, 3. 93*

Curpic, G. $89^{*}$

Curtoni, E. $888^{*}$

Cystathionase 265

Cystathionine 265, 360

Cystathionine synthase 23

Cystic fibrosis of the pancreas $167,446,626$

Cystine 501

Cystinosis 501

Czernichow, P. 53

Dagna-Bricarelli, F. 96*

Dajani, A. S. $406^{*}$

Dallas, J. 374*

Dancis, J. 131

Daughaday, W. H. 398*

Daum, R. 392*

Davenport, L. M. 386*

David, J. R. 378*

Davidson, M. 387*

Davies, P. A. 89*

Davis, C. W. C. 379*
Davis, M. 298

Dayton, P. G. 419*

Dean, D. $386^{*}$

Deavers, S. 193

de Lannoy, C. W. 382*

Delivoria-Papadopoulos, M. 235, 653*

Dell, R. B. 395, * 523

Delvin, E. 392*

Denning, C. R. 390*

Dent, P. B. 181

Dermatoglyphics 64

Desjeux, J. F. 84*

Desmet, V. 704

Desnick, R. J. 83*

Development 17

Developmental biochemistry 70, 101, 151, 281

Developmental enzymology 704

Developmental immunology 539

Developmental physiology 126, 193, 312, $356,472,548,586,698$

De Vos, R. 704

De Wolf-Peeters, C. 704

Diabetes mellitis 605

Diaz-Bensussen, S. 213

Diaz del Castillo, E. 213

Didisheim, P. 409*

Diener, M. M. 17

Dietrich, M. 88*

Dietzman, D. E. 652*

Di George, A. M. 392*

Di Menza, S. 420*

2,3-Diphosphoglyccrate 235

Disaccharides 213

di Sant'Agnese, P. A. 389,*626

Di Stephano, J. J. 399*

DNA 312,329

Doctor-patient relations 298

Dodson, W. E. 501

Donath, A. $86, * 618$

Dorson, W.J. 376*

Drack, E. 167

Draper, D. 413*

Drash, A. L. 394*

Driscoll, S. 426,* 539

I) ubois, J. 86*

Dubois, R. S. $388^{*}$

Dully, M. 424*

Dumpit, F. 414*

Duncan, R. 414*

Dungy, C. $388^{*}$

Dupree, E. 378*

Dupuy, J. M. 88*

Dussault, J. H. 399*

Dyer, N. 373*

Dzierba, J. L. 650*

Eberlein, W. R. 389, ${ }^{*} 400,{ }^{*} 423 *$

Eckerd, J. M. 399*

Eckman, L. N. 404*

Ecological adaptation 691 
Ecology 17

Edelman, R. 380*

Edelman, C. M., Jr. 382, ${ }^{*} 383^{*}$

Eeckels, R. 83*

Egan-Mitchcll, B. 84*

Eggermont, E. 83*

Eisner, G. M. 335

Eitzman, D. V. 427*

Ekelund, H. 91*

Elders, M. J. 398*

Eldjarn, L. 84*

Eller, J. J. 380, * 402*

Embryos 472

Emmanouilides, G. C. $415^{*}$

Enzyme activity 366

Enzyme induction 70

Enzymes 466

Epincphrine 472

Erenberg, A. 415*

Erkan, N. V. $396^{*}$

Erythroblastosis fetalis 683

Ery throcyte 159, 614

Erythrocy te lipids 683

Erythrocytes 101, 356, 446

Escherichia soli 246

Esterly, N. B. 411*

Estradiol $17 \beta 246$

Evans, C. A. N. 92*

Evans, H. E. $411^{*}$

Extracellular fluid 523, 618

Faiman, C. 401*

Fanaroff, A. $411^{*}$

Fat 126

Fatty acids 101,466

Faxclius, G. 373*

Feig, S. A. 409*

Felig, P. 413*

Fernald, G. W. $427^{*}$

Fernandes, J. 633

Ferrieri, P. 406*

Fetal hemoglobin 493

Fetterman, G. H. 385*

Fetus 2, 17, 70, 113, 131, 151, 274, 366 $452,539,586$

Fibrinolysin 206

Fibrinolysis 113

Fibroblast 366, 501

Fine, R. N. 644*

Fingerprints 64

Finnström, O. 89, 91 *

Fisher, D. A. $398,{ }^{*} 399 *$

Fisher, D. E. 415*

Fitzgerald, J. F. 698

Flad, H.-D. 88*

Fletcher, A. P. 655*

Fliedner, T. M. 88*

Florman, A. L. 403*

Flynt, W. J., Jr. 405*

Fog, J. 94*

Foley, T. P., Jr. 398*
Fong, S. W. 377*

Fossati-Gugliclmoni, A. 96*

France, F. L. $411^{*}$

Franks, R. C. $400^{*}$

Frederickson, D. S. 653*

Frce anti-D antibodies $95^{*}$

Free fatty acid 605

Free fatty acids 33

Freeman, R. K. 655*

Freeman, R. B. 385*

Frcemon, B. 298

French, J. 395*

Frenck, N. 91*

Frenk, S. 417*

Friedman, S. B. 638*

Friedman, W. F, 425*

Fructose-1, 6-diphosphatase 633

Früs-Hansen, B. 85*

Fujimoto, W. Y. 77

Fulginite, V. A. $380,{ }^{*} 402 *$

$\beta$-Galactosidase 366

Gall, D. G. $410^{*}$

Gammaglobulin 2

Gardner, J. D. 446

Gardner, L. I. 422*

Garland, J. T. 398*

Gartner, L. M. 413*

Garwicz, S. 94*

Garwood, V. P. 199

Gas trapping 10

Gatti, R. A. 378*

Gaull, G. E. 23, 86, ${ }^{*}$ 265, 392*

Gekle, D. 40)

Gelfand, E. W. 378*

Genetic disease 107, 137, 256, 265, 366, $446,501,511,518,614,633$

Genova, R. 88*

Genscher, U. 88*

Gentz, J. C. H. 435

Gerald, P. S. 64, 645*

Gersh, R. 423*

Gershanik, J. J. 414*

Gestation 586

Gewurz, H. 380*

Ghadimi, H. 393*

Gibbons, I. S. E. 390*

Giebink, G. S. 400*

Gilbert, R. D. 427 *

Giles, J. P. 403,* 649*

Gill, F. M. 409*

Ginsberg-Fellner, F. 392*

Glade, P. R. 378*

Glasgow, J. F. T. 427*

Glasgow, L. A. 403*

Glass, L. $411^{*}$

Glomerular filtration rate 40

Glorieux, F. 392*

Glucagon 33, 126

Gluck, L. 655*

Gluconeogenesis 633
Glucose 33, 159, 518

D-Glucose 356

Glutamate-oxaloacetate transaminase 281

Glycine 478

Glycogen 107

Glycogenosis, type II 107

Gm factor 2

Go, S. C. $411^{*}$

Godwin, H. A. 378*

Gold, R. J. M. 421*

Goldberg, S. J. 425*

Goldman, A. S. 378, * 400*

Goldman, H. 392*

Goldschneider, I. 402*

Goldstein, D. 376*

Good, R. A. $88,{ }^{*} 378,{ }^{*} 380^{*}$

Goodman, D. S. 390*

Gordon, B. A. 137

Gotlin, R. W. 388, ${ }^{*} 400^{*}$

Gotschlich, E. C. 402*

Gottovi, D. 427*

Gracey, M. 84*

Graham, G. G. 691

Grand, R. J. 388*

Grant, D. K. 650*

Grant, J. W. 424*

Grave, G. D. 659

Gray, J. A. 246, 377*

Graystone, J. E. 605

Greenberg, A. H. 53

Greene, M. L. 77

Greenficld, S. 401*

Greensher, A. 423*

Greer, M. 652*

Greifer, I. 382*

Gresham, E. L. 419,*640*

Gretsky, N. E. 374*

Gribetz, I. 380*

Griffith, J. F. 373,* 404*

Grimley, P. M. 501

Grippo, J. 466

Griscom, N. T. 427*

Griswold, W. R. 385*

Gross, G. P. 406*

Grota, L. J. 638*

Grotsky, H. 389*

Growth 33, 173, 691

Growth hormone 33, 605

Growth retardation 59, 312

Grumbach, M. M. 646, *654*

Gruskin, A. B. 392*

Grushkin, C. M. 644*

Guerra, F. A. $381^{*}$

Gustafson, A. 86*

Gutberlet, R. 373*

Guthrie, R. A. 668

Haberman, J. D. 644*

Hahn, P. 126, 412*

Hakkarainen, J. 435

Hall, N. 356 
Hall, R. T. 4l4*

Haltalin, K. C. $404^{*}$

Halvorsen, S. 94*

Hamburger, R. N. $381^{*}$

Hamilton, J. R. 389*

Hamilton, R. 413*

Hammond, J. 649*

Hammond, K. B. 375, * 395*

Hannel, K. H. 96*

Hanson, L. ̊. 89*

Harcke, H. T., Jr. 17

Harrah, J. L. 386*

Harvengt, C. $83^{*}$

Hashim, S. A. 387*

Hathaway, W. E. 395,* 406*

Haust, M. D. 137

Havlicek, V. 428*

Hayslett, J. P. 384*

Head circumference 691

Heagarty, M. 652*

Helmuth, A. C. 151

Hematocrit 193

Hcmoglobin 235

Hemolysis 159

Hengstenberg, F. 394*

Henley, W. L. $381^{*}$

Hentel, J. 424*

Hercz, A. $427^{*}$

Hereditary spherocy tosis 614

Herod, L. 586

Herrin, J. T. $383^{*}$

Herrmann, K. L. 405*

Herz, F. 407*

Hessler, J. R. 427*

Heymann, M. A. 424, * 425,* 452

Higurashi, M. 407*

Hilgartner, M. 408*

Hill, D. E. 312, 418*

Hilleman, M. R. 402*

Hinze, G. 89*

Hirschhorn, K. 424*

Hirsh, J. 409*

Hitzig, W. H. 89, ${ }^{*} 95^{*}$

Hjelm, M. 96*

Hobel, C. J. 415*

Hochapfel, G. 88*

Hodson, W. A. 428,* $429^{*}$

Hoffman, L. E., Jr. 472

Hohenaucr, L. 90*

Holley, K. E. 383*

Holliday, M. A. 384, ${ }^{*} 386 *$

Hollingsworth, D. R. $397,{ }^{*} 399^{*}$

Holmes, B. 380*

Holmes, L. 373*

Holmgren, J. 89*

Holowach, J. 374*

Holt, A. B. 312, 418*

Holtzapple, P. G. 389*

Homocystinuria 23, 265

Homolanthionine 265

Hong, R. 385, ${ }^{*} 655^{*}$
Honig, G. R. 159

Hoogenraad, N. J. 418*

Hormones 126

Hossaini, A. 413*

Howe, R. B. 256

Howic, V. M. 401*

Høyeraal, H. M. 96*

Hsia, Y. E. 395*

Hsu, L. Y. F. 424*

Huang, S.-W. $655^{*}$

Huber, G. 405*

Hug, G. 107, 410, * $421 * 646^{*}$

Fuggins, R. A. 193

Hughes, E. R. 398*

Huisman, T. H. J. 493

Hülsmann, W. C. 633

Humbert, J. R. 395*

Humoral transducibility 167

Hunter's syndrome 137

Hurler's syndrome 137

Hurwitz, R. 388*

Hustu, H. O. 408*

Hypercapnia 523

Hyperglycincmia 478

Hyperheparitinsulfaturia 137

Hyperlysincmia 511

Hypermethioninemia 23

Hyperoxia 659

Hyperphosphatasemia 591

Hypophosphatemia 591

Hypoxanthinc-guanine phosphoribosyltransferase 77

Hypoxia 173

Hyvarinen, M. A. 415,* $416^{*}$

\section{$\operatorname{IgM} 246$}

Immunodeficiency 226

Immunologic development 246

Inanition 59

Infantile malnutrition 33

Infants, 2, 213, 345, 356, 379, 591, 673, 683

Infection 246

Inflammatory response 487

Ingelfinger, J. R. 655*

Insulin 33, 605

Intelligence 579

Intestinal transport 698

In tracellular water 673

In trauterine growth retardation 673

In trauterine transfusion 683

Intravascular coagulation 113

Ion transport 159

Isovaleric acidemia 478

Isracls, E. D. 113

Isracls, L. G. 113, 614

Isselbacher, K. J. 386*

Jackson, S. H. 413*

Jacobs, H. S. 401*

Jaffe, N. 427*

Janncs, E. J. 419, *640*
Jancway, C. A. 560

Jaundice 256

Jehle, J. W. 659

Jellum, E. 84*

Jenner, M. R. 646*

Jéquier, E. $91^{*}$

Jerauld, R. 639*

Jodal, U. 89*

Johnson, $\Lambda$. M. 427*

Johnson, J. 414*

Johnston, R. B., Jr., 401, * 408*

Joncas, J. H. $405^{*}$

Jones, E. M. 392*

Jose, P. А. 335

Joss, E. E. 92*

Julius, R. $380,{ }^{*} 652 *$

Kaas, W. P. 591

Kaback, M. M. 366

Kaijser, B. 89*

Kaiser, D. 167

Kaizer, H. 408*

Kan, Y. W. 407*

Kaplan, E. 407*

Kaplan, S. 426*

Kaplan, S. L. $646,{ }^{*} 654^{*}$

Karaklis, A. 92*

Käser, H. 92*

Kashgarian, M. 384*

Kastenschmidt, L. L. 281

Kattwinkel, J. 389*

Katz, F. H. 400*

Katz, R. M. $381^{*}$

Katz, S. L. 373*

Kaufman, R. L. 416*

Kckonäki, M. 86*

Kelch, R. P. 646*

Kellum, M. 435

Kelly, L. F. X. 396*

Kelly, M. 389*

Kclsch, R. C. 383*

Kennaway, N. G. 287

Kennedy, C. 659

Kenncll, J. 639*

Kenny, F. M. 399*

Kenny, J. F. 246, 377*

Kerr, G. R. 151

Ketoacidemia 518

Ketoacidosis 478

Kidney 40, 213, 591, 618

Kim, H. W. 649*

Kim, Y. J. 413*

King, K. 417*

Kirshner, N. 425*

Kistenmacher, M. L. 654*

Kjellmer, I. 86*

Klaus, M. $411{ }^{*} 639^{*}$

Klein. A. 399*

Klein, R. 410*

Kleinman, L. I. 382, ${ }^{*} 393 *$

Klenk, E. L. 395* 
Klingberg, W. G. 478

Klock, L. E. Jr. 402*

Klossner, J. $91^{*}$

Klujber, L. 93*

Kniker, W. T. 381*

Knittle, J. 392*

Knobloch, H. 376*

Koivikko, $\Lambda .91^{*}$

Kolko, N. 411*

Kontras, S. B. 421*

Kopel, F. B. 389*

Kopelnan, A. E. 642*

Korsch, B. M. 298, 644*

Kouvalaincn, K. 95*

Krauss, $\Lambda$. N. 10

Kreger, N. C. $639^{*}$

Kremer, W. P. 379*

Kretchner, N. 388, ${ }^{*} 418^{*}$

Kretschmer, R. R. 226

Kricger, D. 88*

Kricger, I. 417*

Krivit, W. 83,* 93,*274

Krugman, S. 403, ${ }^{*} 649^{*}$

Kulorich, M. V. 655*

Kumar, S. 393*

Kumar, V. 372, *37.5*

Kutt, H. 408*

Kwashiorkor 579

Labowskic, R. J. 380*

I acson, P. S. 159

Lactate 213

I.actic acid 633

Lactic dehydrogenase 281

I actoferrin 514

Ijanarre, A. 426*

Lamm, P. 392*

I.ampkin, B. C. $410^{*}$

Lanc, 1). $413^{*}$

Lange, K. 384*

Lanman, J. T. 586

r.änsimics, E. $91^{*}$

Iapcy, А. 389,* 446, 626

Lardinois, R. $87^{*}$

Larese, R. 653*

Laster, I. 389*

Lattos, I). 655.

Lavy, U. 387*

Lawton, A. K. $377^{*}$

Lean body mass 673

Lebenthal, E. 388*

Lec, C. E. 523

Leffler, $А$. T., II 518

Lcikin, S. 377,* 404*

Lconard, C. O. 366

Lepow, M. L. 402*

Jerch, P. O. 493

Lesch-Nyhan syndrome 77

Lecukocy tes 487, 501

Levi, H. 374*

Levin, S. $87^{*}$
Levine, R. L. 418*

Levison, H. 426, * 427, * $428 *$

Levkoff, A. H. 414*

Levy, R. A. $425^{*}$

Levy, R. L. 385*

Li, T.-K. $412^{*}$

Liden, G. B. $421^{*}$

Lie, S. O. $85, * 94 *$

Lieberman, E. 644*

Lifshitz, F. 213

Light, G. S. 383*

Light, I. J. 393*

Lilienficld, L. S. 335

Linarelli, L. G. 398*

Lindemann, R. 94*

Lindroos, M. C. 70

Linshaw, M. A. 392*

Lipids 101,435

Lipolysis 126

Litt, I. F. 391, * 641*

Litwin, S. D. 2

Liver 70, 274, 548, 633, 704

Lo, S. S. 95*

Logan, A. G. 335

Lonsdalc, D. 396*

Low birth weight infants 17,101

Lowman, J. T. 406*

Lubin, A. H. $375^{*}$

Lubin, B. 406*

Lucarelli, P. 42:3*

Lukc, K.-H. 409*

Lukcns, J. 410*

Lundak, R. $377^{*}$

Lung 10,586

I.ymphocytc. 5.39

Lymphocytes 226

Lynch, M.J. 427*

Lynch, V. $413^{*}$

Lyon, G. M., Jr. 360

Lysine 511

Lysosonal acid $\alpha$-glucosidase 107

Mabry, C. C. 397, $399 *$

Macaca mulatta 151

MacGillivray, M. 397*

Mackenzie, S. 382*

-2-2-Macroglobulin 206

Madahar, D. P. 375*

Madden, D. L. 652*

Magnesium 131

Magrini, U. 88*

Mahoney, C. P. 385*

Mahoncy, M. J. 395*

Maisels, M. J. 412*

Makowski, E. I. $419,{ }^{*} 640^{*}$

Maldonado, N. 387*

Malnutrition 17, 59, 131, 579

Mamer, O. 392*

Mangos, J. A. 418*

Mangurten, H. H. 415*

Manscll, A. 426,* $428^{*}$
Mapes, C. A. 83*

Marcoux, J. 395*

Margolis, C. Z. $412^{*}$

Marliss, E. B. 397*

Marti, H. R. 95*

Martin, A. 396*

Martin, A. L. A. 396*

Martin, M. M. 396*

Martinez-Garza, V. 213

Martini Mauri, M. 93*

Masera, G. 97*

Massimo, L. $96^{*}$

Matalon, R. 428*

Maternal-fetal incompatibility 2

Maternal-fetal interaction 53

Matoth, Y. 94, ${ }^{*} 96^{*}$

Matsaniotis, N. 95*

Matthew, E. B. 652*

Maturation 335, 493, 6.59

Maurer, H. S. 159

Maurer, H. M. $413^{*}$

May, A. G. 385*

McAdams, A. J. 384*

McAlpine, W. 639*

McCormick, A. Q. 412*

McEnery, P. T. 384*

McIntire, M. S. 376*

McIntosh, R. M. 385*

McNair Scott, T. F. 376*

McNamara, H. 641*

McNicholl, B. 84*

MeSherry, N. R. $418^{*}$

Meconium 274

Méhes, K. 93*

Mchta, A. 426*

Mellman, W. J. 420*

Mclnick, J. I. 403*

Meltzer, S. 399*

Mendicini, M. 85*

Menkes, J. H. 374*

Menkes, J. H. 466

Mental retardation $77,137,287,329,668$ 69

Merler, E. 539

Meschia, G. 419,* 640*

Mestyán, J. 92*

Mctabolic aberrations 366

Mctabolic disease 518

Mctcoff, J. 4.17*

Mcycr, H. B. P. 376*

Meyer, R. A. 426*

Microsomes 466

Migeon, B. R. 420, ${ }^{*} 424 *$

Milk 514

Miller, A. 381*

Miller, D. A. $423,{ }^{*} 424 *$

Miller, L. 653*

Miller, M. E. $379,{ }^{*} 487,655^{*}$

Miller, O. J. $423, * 424 *$

Miller, R. A. $426^{*}$

Milner, R. D. G. 33 
Milstead, R. R. 673

Mininberg, D. T. 416*

Mirkin, B. L. 653*

Mitochondria 23

Modanlou, H. 415*

Moe, P. J. 96*

Monkus, E. 655*

Montoya, F. 416*

Moore, E. S. 382*

Moore, T. J. 356

Mori, P. G. 96*

Morin, C. L. 84*

Morrow, G. 396*

Morton, K. C. $375^{*}$

Moser, H. W. 372*

Moshang, T., Jr. 389, ${ }^{*} 398,{ }^{*} 400,{ }^{*} 423 *$

Mosier, H. D., Jr. 59

Mott, J. C. 93*

Mulay, S. $422^{*}$

Mumford, D. M. 407*

Murphy, J. V. 372*

Murphy, S. 374*

Murthy, D. Y. 397*

Muscle 281

Muscle mass 312

Mussa, G. C. $93^{*}$

Mustafa, M. G. 419*

Myclination 466

Nachman, R. L. 411*

Nadler, H. I.. $421,{ }^{*} 422 *$

Nacye, R. L. 17, 374*

Nagahama, H. 380*

Nahmias, A. J. 405*

Naiman, J. L. 390*

Nakamura, N. 97*

Nash, M. A. 382*

Nathan, D. G. $407,{ }^{*} 409 *$

Nathenson, G. 2, 641*

Nedwich, A. 390*

Ncerhout, R. C. 101, 410, ${ }^{*} 683$

Negrete, V.F. 298

Nelson, J. D. 404*

Nconate 518

Neonates 487,673

Nephrotic syndrome 206

Neu, R. L. 422*

Ncufeld, E. F. 83*

Neuroblastoma 360

Ncuwclt, S. 420*

Newborn 151, 173, 274

Newborns 53, 435, 493, 659

Nissley, P. 398*

Niswander, J. D. 64.

Norepinephrine 435

Normand, I. C. S. 90*

Norton, A. C. 199

Novak, L. P. 383*

Novak, M. $655^{*}$

Nutrition 691

Nuzum, C. T. 394*
Nyhan, W. L. 478

Obesity 605

O'Brien, D. 395*

O'Brien, J. S. 416*

Odell, G. B. 548, 642*

Odell, W. D. $401^{*}$

Oetliker, O. 618

Ogawa, Y. 586

Ogra, P. L. $650^{*}$

Oh, W. $415,{ }^{*} 416^{*}$

Okada, K. $416^{*}$

Okas, S. 381*

O'Leary, J. L. 374*

Olegård, R. 86*

Olin, P. 85*

Oliver, T. K., Jr. 414*

Oliver, W. J. 383*

Olsson, T. 90*

Olver, R. E. 90*

O'Neill, A. 415*

Organ growth 17

Orgel, H. A. 381*

Orsatti, M. 84*

Ortiz, M. 87*

Orzalesi, M. $423,{ }^{*} 426 *$

Orzalesi, M. M. 412*

Osborn, J. E. 409*

Oshin, A. 84*

Oski, F. A. $235,374,{ }^{*} 406,{ }^{*} 647,{ }^{*} 653 *$

Osmosis 159

Osteopetrosis 181

Ostcosclerosis 181

Overall, J. C., Jr. 403*

Owen, C. A., Jr. 409*

Owen, G. M. 375*

Oxygen 659

Oxygen affinity 235

Oxygen consumption 199, 435

Oxygen transport 235

Pabst, H. F. $380^{*}$

Page-El, E. 420*

Pagtakhan, R. D. 428*

Palmarino, R. 423*

Palmar patterns 64

Pande, H. 84*

Pantelakis, S. N. 92*

Parathormone 181

Parathyroid glands 40

Pardo, V. 655*

Parkinson, D. K. $417^{*}$

Parks, C. R. $428 *{ }^{*} 429 *$

Parmenticr, C. M. 428*

Parmley, T. H. 366

Parra, A. 605

Parrott, R. H. 649*

Partin, J. C. $391^{*}$

Pasamanick, B. 376*

Pascual, E. 411*

Pataki, L. 95*

Paton, J. B. 382, ${ }^{*} 415^{*}$
Peakman, D. 423*

Pediatric community 298

Pelkonen, P. 87*

Peller, J. 274

Pérez-Santiago, E. 387*

Persson, B. 435

Persutti, R. 416*

Peter, G. 401*

Peters, B. $386^{*}$

pH 345

Phagocy tosis 487

Phenobarbital 256

Phenolic acids 287

Phenylalanine 329

Philippart, M. 394*

Philippidis, P. 390*

Phosphate reabsorption 40

Phosphaturia 345

Phosphorylase 281

Physiological development 335

Phy tohemagglutinin transformation 539

Piceni Sereni, L. 97*

Picchocki, J. T. 412*

Piel, C. F. 384*

Picro, M. $84^{*}$

Pigram, P. 423*

Pildes, R. S. $420^{*}$

Piñeiro, C. 579

Pinkel, D. P. 408*

Pinsky, L. 422*

Pipecolic acid 511

Placenta 113, 13I, 274, 452

Plasma membrane 356

Plasma volume 193

Plasminogen 206

Plato, C. C. 64

Plenert, W. 88*

Plunket, D. C. $380^{*}, 402 *$

Plüss, H. I. 89*

Polcy, J. R. 390, 644*

Pool, J. G. 412*

Poretti, G. 86*

Portnoy, B. 403*

Potassium 159, 167, 618

Potter, D. E. 384,* 386*

Powell, D. W. 39I*

Pregnancy 131

Premature infant 235

Premature infants 10

Pressor reccptors 472

Price, J. W. 396*

Prod'hom, L. S. 91*

Protein, 173, 329

Protein malnutrition 312

Pruitt, A. W. 419*

Pulmonary function 10

Punnett, H. H. 654*

Pupene, M. B. 511

Purine metabolism 77

Pyridoxine 23

Qazi, Q. H. 375* 
Quic, P. G. 380*

Raddc, I. C. $413,{ }^{*} 417 *$

Rahimtoola, S. H. 426*

Räihä, N. C. R. 70, $86^{*}, 452$

Raivio, K. O. $396^{*}$

Ransome-Kuti, O. 388*

Rasmussen, K. 478

Rasmussen, L. F. 420*

Rassin, D. K. 265

Rathi, M. 393*

Raumaker, D. L. 407*

Raye, J. 373*

Raye, J. R. 419, ${ }^{*} 640 *$

Reade, T. 392*

Reiscr, S. 698

Renal blood flow 335

Renal cortical volume 335

Renal disease, chronic 206

Renin 626

Rennert, O. $380, * 652^{*}$

Renold, A. E. $397^{*}$

Respiratory distress syndrome 235, 345

Reuter, J. H. 382*

Reynolds, E. O. R. 90*

Reynolds, J. 92*

Reynolds, M. 92*

Rezza, E. 85*

Ribble, J. C. $652^{*}$

Richard, G. A. $385,{ }^{*} 386^{*}$

Richards, S. E. M. 381*

Rickets 40

Rickets, vitamin D deficient 591

Rigatto, H. 414*

Rimoin, D. L. $416^{*}$

RNA 329

Robbins, J. B. 514

Roberton, N. R. C. 93*

Roberts, R. B. $652^{*}$

Robinson, A. A. $380,{ }^{*} 423 *$

Robinson, L., Jr. 407*

Robson, A. M. 655*

Rocklin, R. E. $378^{*}$

Rodey, G. 380*

Rodgerson, D. O. 372, ${ }^{*} 388^{*}$

Rodnan, J. B. 393*

Rodriguez-Soriano, J. 382*

Rogers, M. 373*

Romeo, G. 420*

Roncevic, N. P. 235

Roof, B. S. 384*

Rosado, A. 417*

Rosen, F. S. 226, 378*

Rosen, K. M. 426*

Rosenberg, L. E. 394, ${ }^{*} 395^{*}$

Rosenthal, M. D. 419*

Rosenthal, R. 416*

Rossi, E. 167, 618

Rostock, D. 40

Rudolph, A. M. 424,* 425,* 452

Ruuskanen, O. 95*

Ryser, G. 91 *
Sagel, I. $384^{*}$

Saigal, S. $415^{*}$

St. Geme, J. W., Jr. 379*

Salafsky, I. S. $422^{*}$

Saltzman, A. 383*

Sanfilippo disease; mucopolysaccharides 137

Santiago-Borrero, P. J. 387*

Santini, R. $387^{*}$

Sapse, A. T. 381*

Sass-Kortsak, $\Lambda .427^{*}$

Saunders, N. R. 92*

Savilahti, E. 87*

Schaefer, S. J. 375*

Schafer, I. A. $391^{*}$

Schaffer, A. 586

Schaffner, F. 23

Schain, R. J. 173

Schärer, K. 92*

Scheinman, J. I. 206

Schenk, E. A. $385^{*}$

Schcye, E. 407*

Schneidman, K. 265, 392*

Schonauer, T. $655^{*}$

Schonberg, S. K. $391^{*}$

Schorr, J. B. 2

Schrempf, G. 89*

Schretlan, E. D. A. M. 83*

Schroeder, W. A. 493

Schubert, W. K. 107, 391,* 421,*646*

Schulkind, M. 380*

Schulkind, M. L. 379*

Schulman, J. D. 77, 501

Schulte, F. J. 89*

Schultz, R. B. 605

Schultz, S. 618

Schütt, B. 618

Schwartz, A. D. 412*

Schwartz, A. L. 70, 86*

Schwartz, E. 409*

Schwartz, R. 417*

Schwartz, R. H. 385*

Schwieler, G. H. 90*

Scott, C. R. $416^{*}$

Scriver, C. $382,{ }^{*} 392,{ }^{*} 421 *$

Seegmiller, J. E. 77, 396*

Segal, M. B. $92^{*}$

Segal, S. $412^{*}$

Sela, M. 87*

Sell, S. H. 401*

Selstam, U. 90*

Semar, M. 384*

Seppäla, M. 86*

Sereni, F. 97*

Sever, J. L. $652^{*}$

Sey, M. 392*

Shahidi, N. T. 409*

Shamberger, R. J. 396*

Shami, Y. $417^{*}$

Sharp, H. L. 93, ${ }^{*} 274$

Shaw, M. 398*
Shelton, J. B. 493

Shclton, J. R. 493

Shinebourne, E. A. 424,* $425^{*}$

Shira, J. 380*

Shulman, S. T. 405*

Shumway, C. N. 413*

Sialic acid 514

Siassi, B. E. $425^{*}$

Siebcr, O. F., Jr. 402*

Sigstad, H. 93*

Silbcrberg, R. $416^{*}$

Siltzbach, L. E. $378^{*}$

Silverberg, M. 387*

Simonc, J. V. 408*

Simopoulos, A. P. 626

Skeleton 59

Skjaclaaen, P. 94*

Sklenovsky, A. 428*

Skrede, S. 85*

Sloan, H. R. 653*

Slotkoff, L. M. 335

Smith, A. L. $372^{*}$

Smith, C. B. $402^{*}$

Sinith, C. W. 378, ${ }^{*} 390^{*}$

Sinith, D. H. 40 I $^{*}$

Smith, E. I. $390^{*}$

Smith, E. L. 193

Smith, F. R. 390*

Smithwick, E. M. $411^{*}$

Snodgrass, P. J. 394*

Snyderman, R. 404*

Sobcl, B. E. 425*

Sodium 159, 167, 626

Sodium transport 167, 446, 614

Solomon, G. E. 408*

Solomon, S. 422*

Soukup, S. 107, 421,*646*

Spargo, B. H. 384*

Spcnnati, G. F. 423*

Sperling, D. R. 199

Spigland, I. 391*

Spitzer, A. 382*

Splecn 246, 539

Springer, D. 131

Sprinkle, T. $380^{*}$

Spruance, S. L. 402*

Stahlman, M. 373*

Stauffer, U. G. 89*

Stccle, M. W. 393*

Steerman, R. L. 404*

Steffa, M. 639*

Steinberg, S. 403*

Stern, E. R. 374*

Stcvenson, J. 518

Stewart, J. A. 405*

Stickel, D. L. 379*

Stickler, G. B. 383*

Stiehl, A. $391^{*}$

Stichm, E. R. 206, 381, * $416^{*}$

Stockman, J. 374*

Stokes, J., Jr. 402*

Stokke, O. 84* 
Stowers, C. $395^{*}$

Strang, L. B. $90^{*}$

Strauss, J. 199

Strebel, L. 548

Striker, G. E. $385^{\circ}$

Ströder, J. 40

Strómme, J. H. 85*

Stuart, M. 374*

Sturgeon, P. 422*

Sturman, J. A. 86,* 265, 392*

Stutman, O. 88*

Sugarman, H. 653*

Sullivan, J. C. $391^{*}$

Sullivan, M. P. 408*

Sunshine, P. 388, * 414 *

Surainder, Y. 415*

Surfactant 586

Suthcrland, J. M. 393*

Sutow, W. W. 408*

Svenningsen, N. W. 94*

Swanstrom, S. 373*

Swcat 167, 626

Sweelcy, C. C. $83^{*}$

Swerdloff, R. S. 401*

Swyer, P. R. 413*

18q-Syndrome 64

Taber, I. H. 40:3*

Tacusch, W. L. 416*

Talbert, J. L. 312

Tallan, H. H. 26.5

Tantibhedhyangkul, P. 387*

Taussig, H. B. 5699

Taylor, $\Lambda .409^{*}$

'Taylor, H. G. 4.08*

Tedesco, T. A. 420*

Teller, W. 88*

Temperature 173

Teramo, K. A. W. $417, * 452$

Teratology 199

Terrenato, I. 423*

Thaler, M. M. 373,* 391*

Thalidomide 199

Thomaidis, T. 95*

Thompson, R. G. 398*

Thromboembolism 206

Thromboplastin 113

Thurston, D. L. 374*

Thurston, J. H. 392*

Thymus 226, 539

Thyrocalcitonin 668

Thyroid 466

Thyroid gland 181, 668

Thyrotropic hormone 53

Thyroxine 53

Thyroxine-binding globulin 53

Ticknor, W. 414*

Tictzc, H. U. 92*

Tildon, J. T. 394, ${ }^{*} 518$

Ting, R. Y. $376^{*}$

Toca, T. 579

Topilsky, M. 378*
Tori, C. A. 428*

Toro-Sola, M. A. 654*

Torrado, A. 91*

Torrado, A. D. 382*

Trabcrt, R. 396*

Traggis, D. G. 427*

Transfer mechanisms 356

Travis, S. 653*

Trepcl, E. 88*

Treser, G. 384*

Trijbels, J. M. F. 83*

Trueworthy, R. C. $406^{*}$

Tsang, R. C. 393*

Ty, A. 384*

Tyrosine aminotransferase deficiency 287

Tyrosine- $\alpha$-ketoglutarate transaminase 70

Tyrosinemia 287

Tyrosyluria 287

Tyson, J. 53

UDP-glucuronyltransferase 548

Umana, G. 650*

Underwood, B. A. 390*

Ungari, S. 85*

Urmson, J. 374, ${ }^{*} 647 *$

Urrusti, J. 417*

Usher, P. $410^{*}$

Usher, R. 345, 415*

Uy, R. 49:?

Valassi-.1dam, H. 95*

Valdes, O.S. $413^{*}$

Vallet, H. L. 389, ${ }^{*} 396,{ }^{*} 398,{ }^{*} 400,{ }^{*} 423^{*}$

Van Dricssche, R. 83*

Van Ess, J. 38.5*

van Hecswijk, P. J. 83*

Van Mierop, I. H. S. 472

van Munster, P.J.J. 83*

Van Pelt, L. F. 379*

Vapaavuori, E. K. 424, * $425 *$

Velasco, I.. 417*

Vernier, R. L. 654*

Victorin, L. 86*

Vidyasagar, D. 654 *

Villarcjos, V. M. 402*

Vis, H. L. 86*

Visco, G. 399*

Vitamin D 40

Volpe, J. 373*

Waaler, P. E. 84*

Wada, Y. 265

Wagner, I. 376*

Waisman, H. A. 151

Walker, W. A. 386*

Walls, K. W. 405*

Walter, P. 97*

Walton, D. 373*

Wang, N. S. $416^{*}$

Wannamaker, L. W. 406*

Ward, A. N. 478

Warren, I. S. $420^{*}$
Warshaw, J. B. 419*

Wasserman, E. 384*

Watanabe, K. 173

Watkins, W. I. 404*

Weber, H.-P. 591

Wehrle, P. F. 403*

Wei, P. 413*

Weibcl, R. E. 402*

Weiss, L. 424*

Weksberg, R. $422^{*}$

Welcher, D. W. 374*

Wclls, R. 425*

Wennberg, R. P. 42C*

Wertelecki, W. 64, 645*

Werthman, M. W. 412*

West, C. D. $384^{*}$

Whaun, J. M. 647*

Whittemore, R. 426*

Wicsmann, U. N. 83*

Wigglesworth, J. S. 87, * $95 *$

Wilbur, J. R. 407, * 408*

Wilkins, J. $403^{*}$

Williams, C. 652 *

Williams, G. S. 395*

Willians, M. 372*

Williams, R. L. 424, * $425 *$

Willms-Kretschmer, K. 226

Wilson, C. J. 386*

Wincgrad, A. I. 3966*

Winick, M. 420*

Winter, J. S. D. 401*

Winters, R. W. 395, *523

Wohl, M. E. B. 427*

Wolfson, D. R. 514

Wollins, D. G. 137

Wong, K. Y. 410*

Woodrum, D. E. 428,* 429*

Woody, N. C. 511

Woolley, P. V., Jr. 417*

Wright, D. L. 374*

Wu, P. Y. K. $415^{*}$

Yarfe, S. J. 420*

Yakovac, W. C. 389*

Yamazaki, J. 199

Yoshida, P. 417*

Yoshida, T. 417*

Young, L. M. 412*

Younoszai, M. K. 386*

Yow, M. D. 403*

Yuceoglu, A. M. 384*

Zaizor, R. $94,{ }^{*} 96^{*}$

Zarkowsky, H. A. 409*

Zeltzer, P. M. 381,* 416*

Zintl, F. 88*

Zipursky, A. 409, ${ }^{*} 614$

Ziring, P. R. 403*

Zischka, R. 329

Zuppinger, A. 86*

Zuppinger, K. A. 92, * 97 *

Zurbrügg, R. P. 92* 